\title{
Scaling in the BCS to Bose crossover problem in different partial waves
}

\author{
Sadhan K. Adhikari and Angsula Ghosh \\ Instituto de Física Teórica, Universidade Estadual Paulista, 01.405-900 São Paulo, São Paulo, Brazil
}

(Received 15 August 1996)

\begin{abstract}
The BCS superconductivity to Bose condensation crossover problem is studied in two dimensions in $S$, $P$, and $D$ waves, for a simple anisotropic pairing, with a finite-range separable potential at zero temperature. The gap parameter and the chemical potential as a function of Cooper-pair binding $B_{c}$ exhibit universal scaling. In the BCS limit the results for coherence length $\xi$ and the critical temperature $T_{c}$ are appropriate for high$T_{c}$ cuprate superconductors and also exhibit universal scaling as a function of $B_{c}$. [S0163-1829(97)02501-0]
\end{abstract}

A collection of electrons (Fermions), interacting via a weak residual interaction at very low temperature and high density, exhibits pairing instability. Overlapping Cooper pairs ${ }^{1}$ are formed spontaneously according to the BardeenCooper-Schreiffer (BCS) theory of superconductivity. ${ }^{2}$ For usual superconductors, the BCS theory yields $\xi k_{F} \sim 1000$ and $2 \Delta /\left(k_{B} T_{c}\right)=3.52$ in agreement with experiments, where $k_{F}$ is the Fermi momentum, $k_{B}$ the Boltzmann constant, $\Delta$ the gap parameter, $T_{c}$ the critical temperature, and $\xi$ the coherence length.

In the opposite limit of a strong residual interaction, nonoverlapping diatomic bosonic molecules emerge as bound states of fermion pairs. ${ }^{3,4}$ At sufficiently low density and temperature, these composite objects act as an ideal gas of bosons with $\xi k_{F} \sim 0$, which may undergo a phase transition. In three dimensions this phase transition is the usual Bose condensation. ${ }^{3,5}$ In two dimensions one can have a superfluid transition under appropriate conditions. ${ }^{6,7}$ Hence the two extreme limits of the same system exhibit two interesting phenomena.

Leggett ${ }^{3}$ emphasized the importance of the abovementioned BCS superconductivity to the Bose condensation crossover problem. This problem has gained new impetus after the discovery of high- $T_{c}$ cuprate superconductors with certain general properties. ${ }^{5,-10}$ These superconductors have a very small coherence length $\xi k_{F} \sim 10,8,9$ and exhibit a linear scaling between $T_{c}$ and $T_{F}$ known as the Uemura scaling, ${ }^{11}$ where $T_{F}$ is the Fermi temperature. The small value of $\xi$ suggests that its superconducting phase might be understood as one in the above-mentioned crossover regime. $5,7,8,10$

Many high- $T_{c}$ superconductors have a conducting structure similar to a two-dimensional layer of carriers, $8,10,11$ which suggests the use of two-dimensional models. The mathematical complications of the crossover problem are also simpler in two dimensions than in one or three dimensions. ${ }^{5,7-9}$ Hence, in order to understand the subtleties of this problem, the present study is limited to two dimensions.

In the $S$-wave crossover problem in three dimensions, Leggett considered $N$ electrons, each of mass $m$ and of spacing $l$, interacting via a weak short-range potential of range $r_{0}(\ll l)$. The scattering length $a$ satisfies $|a| \gg r_{0}$. By varying the ratio $l / a$, the $\mathrm{BCS}$ and the Bose limits could be attained. When suitably scaled, most of the properties of the system are insensitive to the details of the potential and are universal functions of the dimensionless variable $l / a$. In three dimensions $l \sim k_{F}^{-1}$ and hence one could use the equivalent variable $1 /\left(k_{F} a\right)$. For the $S$-wave problem in two dimensions with a very-short-range attractive potential, the presence of a twobody bound state in vacuum is the necessary and sufficient condition for pairing instability. ${ }^{4,8}$ Hence the convenient dimensionless variable could be $B_{2} / E_{F}$ with $B_{2}$ the two-body binding in vacuum and $E_{F} \equiv k_{B} T_{F} \equiv \hbar^{2} k_{F}^{2} /(2 m)$ the Fermi energy.

The usual treatment of BCS employs a potential in momentum space with a constant value for momentum between $2 m\left(E_{F}-E_{D}\right) / \hbar^{2}$ and $2 m\left(E_{F}+E_{D}\right) / \hbar^{2}$ and zero elsewhere with $E_{D}$ the Debye energy. This implies a moderate range of the interaction. This potential is a physically motivated phonon-induced electron-electron one. ${ }^{2}$ The objective of the present work is to find out to what extent the universal nature $^{4,7,8}$ of the solution of the crossover problem is modified after introducing a smooth potential of medium range in place of the above potential. For some of the high- $T_{c}$ materials, experiments suggest non- $S$-wave Cooper pairing., 8 This is why we have also extended the discussion of universality to the crossover problem in $P$ and $D$ waves, for a simple anisotropic pairing.

The energy $B_{2}$ is not really the ideal variable for studying universality in the crossover problem in two dimensions for non- $S$ partial waves, because in $P$ and $D$ waves one could have Cooper pairing, and hence BCS superconductivity, in the absence of a two-body bound state in vacuum. ${ }^{8}$ The appropriate reference variable for studying the crossover problem in all cases, including the three-dimensional problem, ${ }^{12}$ is the Cooper-pair binding $B_{c}$. For the zero-range $S$ wave model of Ref. $8, B_{2}=B_{c}$. Previously, there have been studies of the crossover problem in terms of the potential strength or scattering length.

We studied the crossover problem with a finite-range separable potential and, especially, the dependence of the universal behavior of the crossover problem on the range of the interaction potential. We found that the universal behavior of the crossover problem does not significantly change with the change of the range of potentials. We calculated the zero-temperature chemical potential $\mu$ and gap parameter $\Delta$ in the entire crossover region, and $\xi$ and $T_{c}$ in the BCS region for different values of the range parameter. We found robust universal scaling in each case, valid over several de- 
cades of Cooper-pair binding. We did not find similar scaling when $\mu, \Delta, T_{c}$, and $\xi$ were considered as a function of potential strength or of binding $B_{2} .{ }^{12}$ The present model also produced an appropriate $T_{c} / T_{F}$ ratio and a small $\xi$ in the BCS region in accordance with experiments on high- $T_{c}$ cuprate superconductors.

The two-body problem and the Cooper and BCS models all exhibit ultraviolet divergences for zero-range potentials and require renormalization to produce finite results. ${ }^{8}$ For non- $S$ partial waves, the nature of these divergences is more complicated and there is no general prescription for renormalization. The present study with finite-range potentials leads to a well-defined mathematical problem without the necessity for renormalization.

We consider a two-body system, each of mass $m$, in the center-of-mass frame. ${ }^{8}$ The single- (two-) particle energy is given by $\epsilon_{q}=\hbar^{2} q^{2} / 2 m\left(2 \epsilon_{q}\right)$, where $q$ is the wave number. We consider the attractive separable short-range potential $V_{p p^{\prime}}=-\lambda f_{p} f_{p^{\prime}}$. The angular momentum $(L)$ dependence of all the variables will not be explicitly shown. This potential leads to pairing instability for any $\lambda, L$, and $f_{p} .{ }^{12}$ In even (odd) partial waves pairing occurs in singlet (triplet) state. The corresponding $t$ matrix is

$$
T_{p p^{\prime}}(2 E)=f_{p} f_{p^{\prime}}\left[-\lambda^{-1}-\sum_{q} f_{q}^{2}\left(2 E-2 \epsilon_{q}\right)^{-1}\right]^{-1},
$$

where $2 E$ is the parametric relative energy. The condition for a bound state at energy $2 E=-B_{2}$ is

$$
\lambda^{-1} \equiv \sum_{q} f_{q}^{2}\left(B_{2}+2 \epsilon_{q}\right)^{-1}
$$

The Cooper-pair problem for two electrons above the filled Fermi sea for this potential is given by ${ }^{1,2}$ $\lambda^{-1}=\Sigma_{q>k_{F}} f_{q}^{2}\left(2 \epsilon_{q}-2 \hat{E}\right)^{-1}$, with Cooper binding $B_{c} \equiv 2 E_{F}-2 \hat{E}$. Using Eq. (1), the Cooper problem is written as

$$
\sum_{q} f_{q}^{2}\left(B_{2}+2 \epsilon_{q}\right)^{-1}-\sum_{q>k_{F}} f_{q}^{2}\left(2 \epsilon_{q}-2 \hat{E}\right)^{-1}=0 .
$$

Leggett ${ }^{3}$ provided a generalization of the BCS model valid for a crossover from large to small coherence lengths at zero temperature. The finite-temperature $(T)$ version of this problem is given by the BCS gap and number equations ${ }^{4}$

$$
\begin{aligned}
& \Delta_{p}=-\sum_{q} V_{p q} \frac{\Delta_{q}}{2 E_{q}} \tanh \frac{E_{q}}{2 k_{B} T}, \\
& N=\sum_{q}\left[1-\frac{\epsilon_{q}-\mu}{E_{q}} \tanh \frac{E_{q}}{2 k_{B} T}\right],
\end{aligned}
$$

with $E_{q}=\left[\left(\epsilon_{q}-\mu\right)^{2}+\left|\Delta_{q}\right|^{2}\right]^{1 / 2}$ and $\mu\left(\neq E_{F}\right)$. At finite temperatures the coupled system of equations (3) and (4) is only valid in the weak-coupling BCS region characterized by positive $\mu / E_{F}$. In the strong-coupling Bose region, characterized by negative $\mu / E_{F}$, due to the existence of preformed composite bosons at finite temperatures above $T_{c}$ the number equation (4) breaks down. ${ }^{5,7}$ Actually, the physical process changes as one moves from the BCS to the Bose limit. In the
BCS limit the formation of Cooper pairs at $T=T_{c}$ signals superconductivity, whereas in the Bose limit the preformed composite bosons may undergo a superfluid phase transition at $T=T_{c}$ under appropriate conditions. ${ }^{6,7}$ At zero temperature Eqs. (3) and (4) are valid in the whole crossover region. ${ }^{8}$

As in Ref. 8, we consider the simplest case of anisotropic pairing in two dimensions where $\Delta_{q}$ has the angular dependence $\sim \exp (i L \theta)$. With the present potential, $\Delta_{q}$ of Eq. (3) then behaves as $\Delta_{q} \equiv \Delta f_{q} \exp (i L \theta)$. Equation (3) then becomes

$$
\lambda^{-1}=\sum_{q} f_{q}^{2} \frac{1}{2 E_{q}} \tanh \frac{E_{q}}{2 k_{B} T},
$$

where $E_{q}=\left[\left(\epsilon_{q}-\mu\right)^{2}+\Delta^{2} f_{q}^{2}\right]^{1 / 2}$. Using Eqs. (1) and (2), Eq. (5) can be rewritten as

$$
\sum_{q>k_{F}} \frac{f_{q}^{2}}{\epsilon_{q}-\hat{E}}-\sum_{q} \frac{f_{q}^{2}}{E_{q}} \tanh \frac{E_{q}}{2 k_{B} T}=0 .
$$

Equation (6) is valid independent of the existence of a twobody bound state in vacuum.

Equations (4) and (6) can be explicitly written as

$$
\begin{gathered}
\int_{E_{F}}^{\infty} d \epsilon_{q} \frac{f_{q}^{2}}{\epsilon_{q}-\hat{E}}-\int_{0}^{\infty} d \epsilon_{q} \frac{f_{q}^{2}}{E_{q}} \tanh \frac{E_{q}}{2 k_{B} T}=0, \\
\int_{0}^{\infty} d \epsilon_{q}\left[1-\frac{\epsilon_{q}-\mu}{E_{q}} \tanh \frac{E_{q}}{2 k_{B} T}\right]=2 E_{F} .
\end{gathered}
$$

Equations (7) and (8) permit the following analytic solutions for $S$-wave $(L=0)$ zero-range potential given by $f_{q}=1$. At $T=0, \Delta=\left(2 B_{c} E_{F}\right)^{1 / 2}$ and $\mu=E_{F}-B_{c} / 2{ }^{8}$ At $T=T_{c}(\Delta=0)$ in the BCS limit $\left(\mu \approx E_{F}\right)$ we find the analytic solutions $\mu+2 k_{B} T_{c} \ln \left[2 \cosh (\mu) /\left(2 k_{B} T_{c}\right)\right]=2 E_{F} \quad$ and $T_{c} / T_{F}=\sqrt{2} \exp (\gamma)\left(B_{c} / E_{F}\right)^{1 / 2} / \pi$ where $\gamma=0.57722$. In this case $2 \Delta /\left(k_{B} T_{c}\right)=2 \pi / \exp (\gamma) \approx 3.528$.

Next Eqs. (7) and (8) are solved numerically in $S$ ( $L=0), P(L=1)$, and $D(L=2)$ waves with form factors $f_{q}=q^{L}\left[\alpha /\left(q^{2}+\alpha\right)\right]^{(L+1) / 2}$ where $\alpha$ is the range parameter. We studied the crossover problem in the entire domain at $T=0$ and calculated the dimensionless order parameter $D \equiv \Delta / E_{F}^{(1-L / 2)}$ and the chemical potential $\mu / E_{F}$ as functions of $B_{c} / E_{F}$ for different $L$ and $\alpha$. The order parameters are shown in Figs. 1(a), 1(b), and 1(c) for $S, P$, and $D$ waves, respectively. In Fig. 2 we exhibit $\mu / E_{F}$ in these cases. The coherence length, or the pair size in the BCS region, defined by $\xi^{2}=\left\langle\psi_{q}\left|r^{2}\right| \psi_{q}\right\rangle /\left\langle\psi_{q} \mid \psi_{q}\right\rangle$, with the zero-temperature pair wave function $\psi_{q}=\Delta_{q} /\left(2 E_{q}\right)$, was numerically calculated using $r^{2} \equiv-\nabla_{q}^{2}$. The calculated $\left(\xi k_{F}\right)^{2}$ are shown in Fig. 3 as a function of $B_{c} / E_{F}$. The $S$-wave Pippard coherence length $\left[=\hbar k_{F} /(\pi m \Delta)\right]$ is also shown in Fig. 3 for comparison. We also calculated $T_{c}$ in the BCS domain by setting $\Delta_{q}=0$ in Eqs. (7) and (8). The calculated $T_{c} / T_{F}$ is also plotted in Fig. 1 for different partial waves.

Before presenting a discussion of the results we mention two limitations of the present model. First, for non- $S$ waves the zero-range limit $(\alpha \rightarrow \infty)$ cannot be taken because of the appearance of strong ultraviolet divergences. For $S$ waves, this limiting solution is analytically known. ${ }^{8}$ Second, the size 

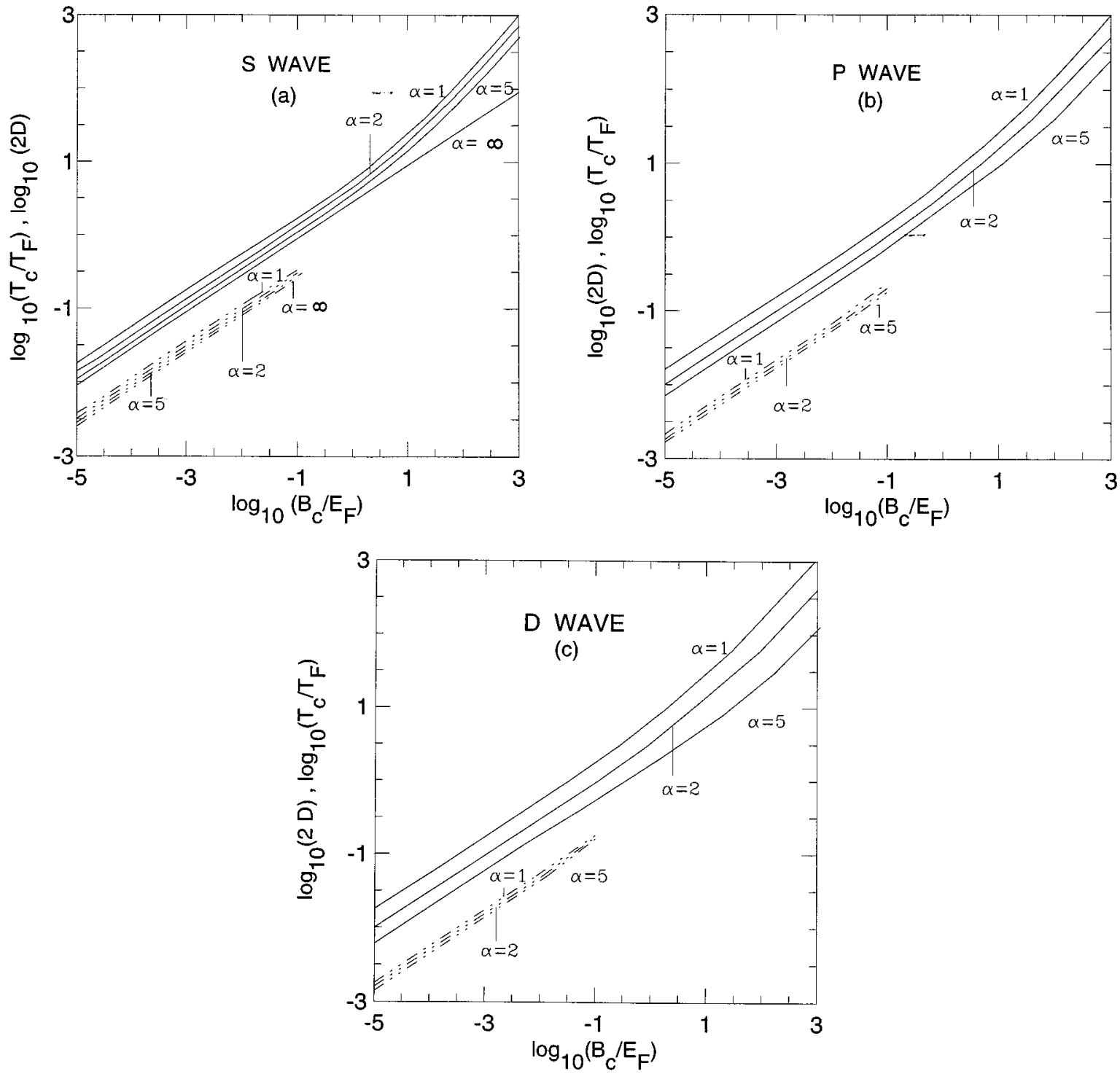

FIG. 1. $\log _{10}$ (2D) and $\log _{10}\left(T_{c} / T_{F}\right)$ vs $\log _{10}\left(B_{c} / E_{F}\right)$ plots for (a) $S(L=0)$, (b) $P(L=1)$, and (c) $D(L=2)$ waves, denoted by solid and dash-dotted lines, respectively, where $D=\Delta / E_{F}^{(1-L / 2)} . T_{c}$ is calculated in the weak-coupling or the BCS regime as the finite-temperature version of the Leggett equations are valid only in this regime.

of the two-body bound state in vacuum with this potential behaves as $B_{2}^{-1 / 2}\left(\alpha^{-1}\right)$ when $B_{2} / 2<(>) \alpha^{2}$. Hence the size of this bound state as well as the Cooper pair becomes unrealistic for $B_{2} / 2>\alpha^{2}$. Consequently, for very large $B_{c} / E_{F}$, the ideal Bose limit of nonoverlapping bosons is not realized for small $\alpha$. Hence the present study is limited to a potential of intermediate ranges $\alpha / E_{F} \sim 1-10$.

From Fig. 1 we find that both $D$ and $T_{c} / T_{F}$ exhibit universal behavior as functions of $B_{c} / E_{F}$ in different partial waves. In the BCS region they exhibit linear scaling valid over about four to five decades. The scaling exponents are roughly constant for all $L$ and $\alpha: D \sim\left(B_{c} / E_{F}\right)^{1 / 2}$, $T_{c} / T_{F} \sim\left(B_{c} / E_{F}\right)^{1 / 2}$. For an ideal Bose gas there is no condensation in two dimensions and hence one might think that $T_{c}$ should reduce to zero as $B_{c} / E_{F}$ increases in the Bose region. However, because of a weak residual interaction between bosons, this system may undergo a superfluid transition with quasi-long-range order below a fixed small $T_{c} / T_{F}$ independent of $B_{c} / E_{F} \cdot{ }^{7}$ The $T_{c}$ for this transition can only be found in numerical model studies. Hence all $T_{c} / T_{F}$ of Fig. 1 should reduce quickly and attain a small constant value as $B_{c}$ increases in the Bose region. ${ }^{7}$ We find that both $T_{c} / T_{F}$ and $D$ increase with decreasing $\alpha$ and $L$. The ratio $2 D /\left(T_{c} / T_{F}\right) \equiv 2 \Delta E_{F}^{L / 2} /\left(k_{B} T_{c}\right)$ increases as $\alpha$ decreases and/or $L$ increases. For example, for $\alpha / E_{F}=1(2,5)$ this ratio is $4.93(4.31,3.87)$ for $L=0,7.07(5.29,4.21)$ for $L=1$, and $10.0(6.37,4.39)$ for $L=2$. The corresponding universal gap-to- $T_{c}$ ratio $2\left|\Delta_{k_{F}}\right| /\left(k_{B} T_{c}\right)$ for $\alpha / E_{F}=1(2,5)$ is $3.50(3.52,3.53)$ for $L=0,3.54(3.53,3.51)$ for $L=1$, and $3.53(3.47,3.35)$ for $L=2$.

From Fig. 2 we find that the zero-temperature $\mu$ has a linear dependence on $B_{c}$ for all $\alpha$ and $L$ almost over the entire crossover region. The minor deviation from linearity occurs for small $\mu$. We present this dependence up to $B_{c} / E_{F}=40$. For larger $B_{c} / E_{F}, \mu$ is essentially given by the zero-range analytic $S$-wave solution: $\mu=E_{F}-B_{2} / 2$.

From Fig. 3, for all $\alpha$ and $L$, we have the universal scaling $\left(\xi k_{F}\right)^{2} \sim\left(B_{c} / E_{F}\right)^{-1}$ valid over three decades of $B_{c}$ in 


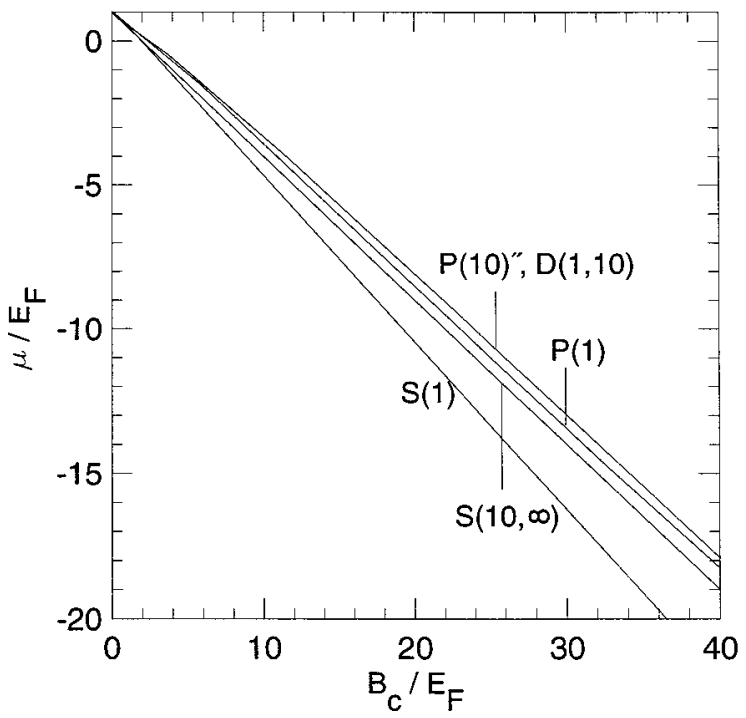

FIG. 2. $\mu / E_{F}$ vs $B_{c} / E_{F}$ plots for different partial waves and $\alpha$. The curves are labeled by partial wave(s) and $\alpha$.

the BCS domain. The corresponding $S$-wave Pippard coherence length satisfies the same scaling. The parameter $\left(\xi k_{F}\right)$ decreases as $L$ and/or $\alpha$ decreases. The analytic zero-range $S$-wave solution has this scaling. ${ }^{8,9}$ For a fixed $B_{c} / E_{F}$, Fig. 1 leads to a scaling of $T_{c}$ with $T_{F}$ for all $\alpha$ and $L$. This scaling was observed by Uemura et al. ${ }^{11}$ for high- $T_{c}$ superconductors. From Fig. 1 we find for $S, P$, and $D$ waves that Uemura's experimental value $T_{c} / T_{F} \simeq 0.05$ leads to a $B_{c} / E_{F}$ in the domain 0.01-0.001, which implies the weakcoupling BCS limit. From Fig. 1 we find that, for $\alpha / E_{F}=5, T_{c} / T_{F}=0.05$ leads to $B_{c} / E_{F}=0.0032(0.0079,0.0135)$ for $S(P, D)$ waves. From Fig. 3 the above Cooper-pair bindings give $\xi k_{F} \simeq 9$ in all partial waves. This implies a universal correlation between $T_{c} / T_{F}$ and $\xi k_{F}$ in all partial waves.

In conclusion, we studied the BCS superconductivity to Bose condensation crossover problem in different partial waves for a finite-range separable potential. We found robust scaling relations involving the order parameter, chemical potential, coherence length and critical temperature as a function of Cooper-pair binding. In the BCS domain the present

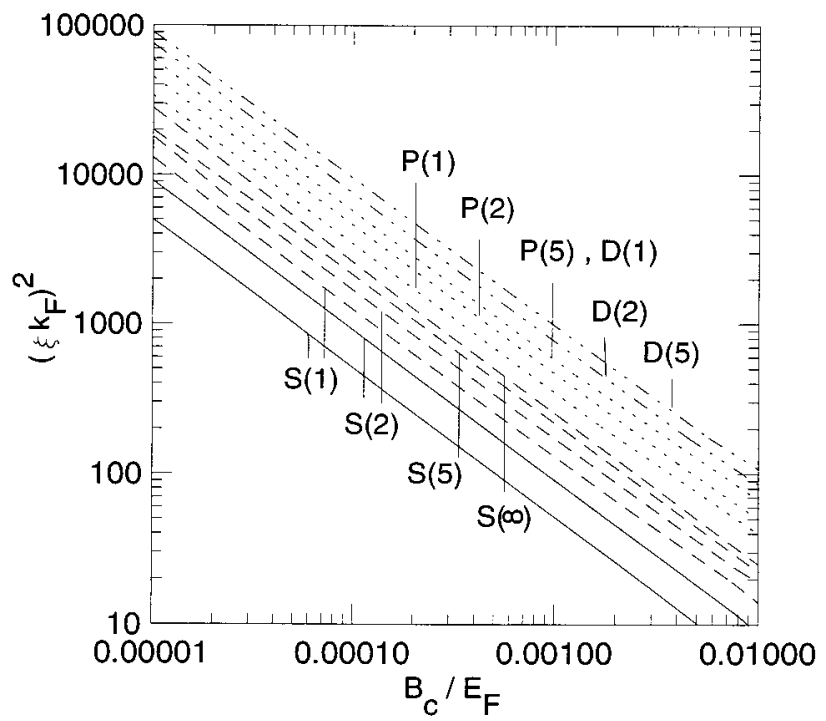

FIG. 3. $\left(\xi k_{F}\right)^{2}$ vs $B_{c} / E_{F}$ plots for different partial waves and $\alpha$ : dashed lines, $S$ - $(L=0)$ wave results; dotted lines, $P-(L=1)$ wave results; dash-dotted lines, $D$ - $(L=2)$ wave results; and solid lines, the $S$-wave Pippard coherence lengths. The curves are labeled by partial wave(s) and $\alpha$.

results may simulate typical high- $T_{c}$ values for the coherence length $\xi$ and $T_{c}$. They also exhibit the $T_{c}$ versus $T_{F}$ linear correlation (at a fixed $B_{c} / E_{F}$ ) as observed by Uemura et al. ${ }^{11}$ The consequence of these findings in describing the high- $T_{c}$ superconductors in two dimensions is not all too obvious. Though we have exhibited the results for a specific separable potential model, we verified that the general trend is maintained as form factors are changed. Hence we do not believe our findings to be so peculiar as to have no general validity. A preliminary study of the $S$-wave threedimensional crossover problem employing the same separable potential as a function of $B_{c} / E_{F}$ also leads to similar universal scaling. ${ }^{12}$ A detailed account of that will be reported elsewhere.

We thank Dr. M. de Llano and Dr. T. Frederico for helpful discussions and Conselho Nacional de Desenvolvimento Científico e Tecnológico, Fundação de Amparo à Pesquisa do Estado de São Paulo, and John Simon Guggenheim Memorial Foundation for financial support.
${ }^{1}$ L. N. Cooper, Phys. Rev. 104, 1189 (1956).

${ }^{2}$ J. Bardeen, L. N. Cooper, and J. R. Schrieffer, Phys. Rev. 108, 1175 (1957); J. R. Schrieffer, Theory of Superconductivity (Benjamin, New York, 1964).

${ }^{3}$ A. J. Leggett, J. Phys. (Paris) Colloq. 41, C7-19 (1980).

${ }^{4}$ K. Miyake, Prog. Theor. Phys. 69, 1794 (1983).

${ }^{5}$ P. Nozières and S. Schmitt-Rink, J. Low Temp. Phys. 59, 195 (1985); R. Haussmann, Z. Phys. B 91, 291 (1993).

${ }^{6}$ J. M. Kosterlitz and D. J. Thouless, J. Phys. C 6, 1181 (1973).
${ }^{7}$ M. Drechsler and W. Zwerger, Ann. Phys. (Leipzig) 1, 15 (1992).

${ }^{8}$ M. Randeria, J-M. Duan, and L-Y. Shieh, Phys. Rev. Lett. 62, 981 (1989); Phys. Rev. B 41, 327 (1990).

${ }^{9}$ M. Casas et al., Phys. Rev. B 50, 15945 (1994).

${ }^{10}$ C. A. R. Sá de Melo, M. Randeria, and J. R. Engelbrecht, Phys. Rev. Lett. 71, 3202 (1993).

${ }^{11}$ Y. J. Uemura et al., Phys. Rev. Lett. 66, 2665 (1991).

${ }^{12}$ A. Ghosh and S. K. Adhikari (unpublished). 